\title{
Quasi-normal modes of static spherically symmetric black holes in $f(R)$ theory
}

\author{
Sayak Datta ${ }^{1, \mathrm{a}} \mathbb{O}$, Sukanta Bose $^{1,2, \mathrm{~b}}$ \\ ${ }^{1}$ Inter-University Centre for Astronomy and Astrophysics, Post Bag 4, Ganeshkhind, Pune 411 007, India \\ 2 Department of Physics and Astronomy, Washington State University, 1245 Webster, Pullman, WA 99164-2814, USA
}

Received: 25 November 2019 / Accepted: 9 December 2019 / Published online: 9 January 2020

(C) The Author(s) 2020

\begin{abstract}
We study the quasi-normal modes (QNMs) of static, spherically symmetric black holes in $f(R)$ theories. We show how these modes in theories with non-trivial $f(R)$ are fundamentally different from those in general relativity. In the special case of $f(R)=\alpha R^{2}$ theories, it has been recently argued that iso-spectrality between scalar and vector modes breaks down. Here, we show that such a break down is quite general across all $f(R)$ theories, as long as they satisfy $f^{\prime \prime}(0) /\left(1+f^{\prime \prime}(0)\right) \neq 0$, where a prime denotes derivative of the function with respect to its argument. We specifically discuss the origin of the breaking of isospectrality. We also show that along with this breaking the QNMs receive a correction that arises when $f^{\prime \prime}(0) /\left(1+f^{\prime}(0)\right) \neq 0$ owing to the inhomogeneous term that it introduces in the mode equation. We discuss how these differences affect the "ringdown" phase of binary black hole mergers and the possibility of constraining $f(R)$ models with gravitational-wave observations. We also find that even though the iso-spectrality is broken in $f(R)$ theories, in general, nevertheless in the corresponding scalar-tensor theories in the Einstein frame it is unbroken.
\end{abstract}

\section{Introduction}

Recent observations of gravitational wave (GW) signals in LIGO and Virgo from compact object mergers have opened up a new chapter in the history of physics [1-4]. So far these detectors have already observed several binary black hole mergers and a binary neutron star merger [5]. The latter observation has put strong constraints on the equation of state of the matter inside neutron stars [6-10]. It has also provided a strong bound on the graviton mass [11] and the deviation of the velocity of GWs from that of the light $[5,12]$. As a result it has already helped us understand not only the merg-

\footnotetext{
a e-mail: skdatta@iucaa.in

be-mail: sukanta@iucaa.in
}

ers themselves but also fundamental aspects of the nature of gravity [12-17]. This scenario is expected to get even more interesting when LISA is launched in several years from now since the space detector will give us the opportunity to test gravity in a different frequency band [18-22].

To date general relativity (GR) has been very successful in observational tests of its predictions (see, e.g., Refs. $[23,24])$. However, certain aspects of GR, e.g., black hole and cosmological spacetime singularities, have invited proposals for higher-curvature correction terms to the EinsteinHilbert action [25-33] in attempts toward resolving these pathologies. These corrections come in various forms, such as functions of Ricci scalars and different combinations of the contractions of the Riemann tensor. These suggestions have gained importance due to other reasons as well. It has been shown that several actions with correction terms can drive inflation and others have been successful in explaining the late time acceleration of the universe [34-37]. Such actions have also arisen from the low-energy limit of quantum corrections or string theory [38-40] and in Loop Quantum Gravity $[43,44]$. These results have initiated extensive research in such alternative theories of gravity in the last few decades. Several studies have already investigated modifications in QNMs for other types of corrections (quadratic in curvature) [41,42]. Now the availability of GW observations helps us examine if these theories can be subjected to useful tests.

As has been shown in numerical relativity, when the two black holes in a binary system merge, a distorted black hole is created. This remnant radiates GWs as it settles down into a Kerr black hole [45-49]. Asymptotically, these GWs can be expressed as superpositions of damped sinusoidal modes, termed as quasi normal modes (QNMs). These QNMs in GR depend only on a couple of parameters characterizing the black hole, namely, its mass and spin, for astrophysical black holes. This is a manifestation of the No-Hair theorem [50-52]. Therefore, observation of these QNMs in the future 
is an anticipated testing ground for the No-Hair theorem and alternative theories of gravity.

It is well known that in GR there are two modes that contribute to GW observables. These are the odd (vector) and even (scalar) modes, and they share the net emitted gravitational energy equally. It has been argued in Refs. [53,54] that in the case of the $\left(R+\alpha R^{2}\right)$ action, where $R$ is the Ricci scalar, QNMs emitted from black holes will not have the same spectrum as in GR. In this work, we show that such a break down in iso-spectrality is quite general across all $f(R)$ theories, as long as they satisfy $f^{\prime \prime}(0) /\left(1+f^{\prime}(0)\right) \neq 0$, where a prime denotes derivative of the function with respect to its argument. We specifically discuss the origin of the breaking of isospectrality. We also show that along with this breaking the QNMs receive a correction that arises when $f^{\prime \prime}(0) /\left(1+f^{\prime}(0) \neq 0\right.$ owing to the inhomogeneous term that it introduces in the mode equation. We also discuss the resulting structural modification of the QNMs.

In an important piece of work, Tattersall et al. [55,56] demonstrated that in Horndeski theory of gravity the QNM equation acquires a source term, which results in the breaking of iso-spectrality. Going by these results alone, one might expect that when an $f(R)$ theory is related to an STT through a conformal transformation, and both theories have the Schwarzschild solution, then iso-spectrality should break in that $f(R)$ theory as well for QNMS in that solution. Interestingly, and perhaps somewhat counterintuitively, we show here that when an $f(R)$ theory and its conformally related STT both have the Schwarzschild metric as solution, and the value of the conformal factor relating these respective solutions is unity, then isopectrality is not broken for the Schwarzschild QNMs in STT even though it is broken for the Schwarzschild QNMs in $f(R)$.

In Sect. 2 general results of an arbitrary $f(R)$ theory and the modified Einstein equations in such theories are discussed. In Sect. 3 we present the perturbation equations in the context of GR around the Schwarzschild metric. In Sect. 4 we discuss the perturbation of the modified Einstein equation in general $f(R)$ theories. In Sect. 4.1 we deduce the different modes and the final perturbation equations in $f(R)$ theories. Then in Sect. 5 we deduce the changes in the QNMs in comparison to GR. Finally, in Sect. 7 we summarize our results. Throughout the work we have adopted the $\{-,+,+,+\}$ signature and set $G=c=1$.

\section{General equations for $f(R)$ theories}

As mentioned above, here we focus our attention on $f(R)$ theories of gravity. Our primary motivation for doing so is that they offer resolutions for certain limitations in the standard cosmological model and the fact that $f(R)$ terms arise as correction terms from loop quantum gravity. Another rea- son is that these theories are not affected by the Ostrogradsky instability [57]. Among all the $f(R)$ theories we only focus on those that allow $R=0$ solutions, for the obvious reason that such solutions exist in GR and are observationally relevant. We discuss this aspect in more detail later.

Let us begin by considering the gravity model that has a Lagrangian of the following form:

$S_{f}=\frac{1}{2 \kappa^{2}} \int d^{4} x \sqrt{-g} f(R)$,

where $\kappa^{2}=8 \pi$. Varying this action with respect to the metric $g_{\mu \nu}$ yields the equation of motion [58],

$R_{\mu \nu} f^{\prime}(R)-\frac{1}{2} f(R) g_{\mu \nu}+\left(g_{\mu \nu} \square-\nabla_{\mu} \nabla_{\nu}\right) f^{\prime}(R)=0$.

It is known that Eq. (2) has a constant curvature solution, $R=\bar{R}$. In that case, the above equation takes the form

$\bar{R}_{\mu \nu} f^{\prime}(\bar{R})-\frac{1}{2} g_{\mu \nu} f(\bar{R})=0$.

Taking its trace gives

$\bar{R} f^{\prime}(\bar{R})-2 f(\bar{R})=0$.

Since our objective here is to study perturbations of the Schwarzschild black hole, we focus only on such models of $f(R)$ that have $\bar{R}=0$ as a root of Eq. (4). It is straightforward to verify that GR is but one example of such theories.

Since our main goal is to look for deviations from GR, we will separately track the GR part of the $f(R)$ action, namely, the $R$ term. Therefore, we will express the Lagrangian as

$f(R)=R+\varphi(R)$,

where $\varphi(R)$ denotes terms in the gravity action beyond GR. Henceforth, we rename $\varphi(R)$ as $f(R)$. Thus, our working Lagrangian becomes $R+f(R)$.

\section{QNMS in general relativity}

For the purpose of our calculations we adopt the gauge invariant formalism discussed in Refs. [59-62]. Our focus remains the Schwarzschild spacetime. In the case of a spherically symmetric spacetime, it is possible to split the manifold into an orbit space and a unit sphere. We will be focusing on the four-dimensional non-spinning black hole background. In that case the manifold splits into a two-dimensional orbit space and a two-sphere. We will use the co-ordinates $x^{a}$ for the orbit space and $z^{A}$ for the two-sphere. The covariant derivatives on the orbit space and the two-sphere are represented by $D_{a}$ and $D_{A}$ respectively. The d'Alembertian operators on the orbit space and the two-sphere are represented by $\tilde{\square}$ and $\hat{\square}$, respectively. 
With proper choice of co-ordinates it is possible to cast the metric in the following form,

$d \bar{s}^{2}=-g(r) d t^{2}+\frac{1}{g(r)} d r^{2}+r^{2} d \Omega^{2}$,

where the overbar represents quantities of the unperturbed background, $g(r)=1-\frac{2 M}{r}$, and $d \Omega^{2}$ represents the metric on the unit two-sphere. The metric perturbation on the background can be expressed as follows [59-62]:

$d s_{p}^{2}=h_{a b} d x^{a} d x^{b}+2 h_{a B} d x^{a} d z^{B}+h_{A B} d z^{A} d z^{B}$,

where $d s_{p}^{2}$ represents the perturbed part of the line element.

To simplify the problem, the metric functions are usually expanded in spherical harmonic basis. After linearizing the Einstein equations, they can be cast in terms of the coordinates of the orbit space alone. This problem has been well studied. We follow the formalism defined by Kodama, Ishibashi and Seto (KIS). With proper identification of the scalar and vector mode it is possible to show that for each multipole $l \geq 2$ they satisfy the following equations [59-61]:

$$
\begin{aligned}
& \frac{d^{2} \Phi_{S}^{G R}}{d r_{*}^{2}}+\left(\omega^{2}-V_{S}\right) \Phi_{S}^{G R}=0, \\
& \frac{d^{2} \Phi_{V}^{G R}}{d r_{*}^{2}}+\left(\omega^{2}-V_{V}\right) \Phi_{V}^{G R}=0 . \\
& V_{S / V}=W^{2} \mp \frac{d W}{d r_{*}}-\frac{\lambda^{2}(\lambda+1)^{2}}{9 M^{2}}, \\
& W=\frac{6 M(2 M-r)}{r^{2}(6 M+2 \lambda r)}-\frac{\lambda(\lambda+1)}{3 M},
\end{aligned}
$$

where $\lambda=\frac{(l-1)(l+2)}{2}$ and the time dependence has been taken to be $e^{-i \omega t}$. The subscripts $S$ and $V$ represent the scalar mode (Zerilli mode) and the vector mode (Regge-Wheeler mode), respectively. Moreover, "GR" in the superscript distinguishes these modes from those in $f(R)$.

These QNMs have been studied extensively in the literature. It has been shown that the transmission and the reflection coefficients of the $V_{S}$ and $V_{V}$ are equal. They make equal contributions to GWs asymptotically. It has also been demonstrated that they share the same frequency spectrum [49]. We will show below that this does not hold, in general, in $f(R)$ theories.

\section{Perturbation in general $f(R)$}

Our main goal is to investigate the QNM structure of the Schwarzschild black hole. Therefore, we take the background metric to be that given in Eq. (6). Now the perturbed metric can be written as

$g_{\mu \nu}=\bar{g}_{\mu \nu}+h_{\mu \nu}$, where $\bar{g}_{\mu \nu}$ and $h_{\mu \nu}$ represent the background metric and its perturbation, respectively. Using the perturbed metric from Eq. (10) in the equation of motion Eq. (2), we find the equation for the perturbation as follows,

$$
\begin{gathered}
\left(1+f^{\prime}(\bar{R})\right) \delta R_{\mu \nu}(h)-(\bar{R}+f(\bar{R})) \frac{h_{\mu \nu}}{2}+f^{\prime \prime}\left[\bar{g}_{\mu \nu} \bar{\square}\right. \\
\left.+\frac{\bar{R}}{4} \bar{g}_{\mu \nu}-\bar{\nabla}_{\mu} \bar{\nabla}_{\nu}-\frac{\left(1+f^{\prime}(\bar{R})\right)}{2 f^{\prime \prime}(\bar{R})} \bar{g}_{\mu \nu}\right] \delta R(h)=0,
\end{gathered}
$$

where $\delta R_{\mu \nu}$ and $\delta R$ are the perturbed Ricci tensor and Ricci scalar, respectively. Equation (11) can be re-expressed as

$\delta G_{\mu \nu}^{e f f}=\kappa^{2} \delta T_{\mu \nu}^{e f f}$,

where

$$
\begin{aligned}
& \delta G_{\mu \nu}^{e f f}=\left[\delta \bar{R}_{\mu \nu}-\frac{1}{2} \bar{g}_{\mu \nu} \delta R\right]-\frac{(\bar{R}+f(\bar{R}))}{\left(1+f^{\prime}(\bar{R})\right)} \frac{h_{\mu \nu}}{2}, \\
& \delta T_{\mu \nu}^{e f f}=-\frac{f^{\prime \prime}}{\kappa^{2}\left(1+f^{\prime}(\bar{R})\right)}\left[\bar{g}_{\mu \nu} \bar{\square}+\frac{\bar{R}}{4} \bar{g}_{\mu \nu}-\bar{\nabla}_{\mu} \bar{\nabla}_{\nu}\right] \delta R(h) .
\end{aligned}
$$

Taking the trace of Eq. (11) it can be shown that

$$
\begin{aligned}
& 3 f^{\prime \prime} \bar{\square} \delta R-\left(1+f^{\prime}(\bar{R})\right) \delta R-(\bar{R}+f(\bar{R})) \frac{h}{2} \\
& +f^{\prime \prime} \bar{R} \delta R=0,
\end{aligned}
$$

where $h$ is the trace of the perturbation. As we show more explicitly below this equation contains a massive scalar degree of freedom that is generically present in $f(R)$ theories, unlike general relativity. Further details are available in Refs. [63-65]. This equation is equivalent to the equation of a massive scalar field with a source term that depends on the perturbation.

\subsection{QNMs in $f(R)$}

In the present work our main goal is to find the perturbed equation of Schwarzschild like solutions in $f(R)$. For that reason we can fix $\bar{R}=0$ along side Eq. (4). This translates to $f(0)=0$. Under these conditions Eq. (14) becomes

$3 f^{\prime \prime}(0) \bar{\square} \delta R-\left(1+f^{\prime}(0)\right) \delta R=0$.

This equation is a source-free massive scalar equation, with $\delta R$ identified as the scalar field and the mass-squared being $m^{2} \equiv \frac{1+f^{\prime}}{3 f^{\prime \prime}}$. Henceforth, whenever there is no scope for ambiguity, we will omit the 0 from the argument value of $f$ and all its derivatives. This massive scalar longitudinal mode $[66,67]$ is absent in GR.

Now the perturbed Einstein equation takes the following form:

$\delta G_{\mu \nu}=\kappa^{2} \delta T_{\mu \nu}^{e f f}$, 
accompanied by the simplified

$\delta T_{\mu \nu}^{e f f}=-\frac{\beta}{\kappa^{2}}\left[\bar{g}_{\mu \nu} \bar{\square}-\bar{\nabla}_{\mu} \bar{\nabla}_{\nu}\right] \delta R$,

where $\beta=\frac{f^{\prime \prime}}{1+f^{\prime}}$. Heretofore, we will use both expressions interchangeably. The important thing to note is that there are possible $f(R)$ theories, such as $\alpha R^{3}$, for which $f^{\prime \prime}=0$ (at $R=0$ ); in that case $\beta$ becomes zero. Another key point is that the information of a particular $f(R)$ theory enters only through the $\beta$. So, apart from $\beta$, dynamically speaking, all $f(R)$ theories are the same.

Now we use the separation of variables to isolate the angular dependence. For this reason we write the perturbed Ricci scalar as $\delta R=\frac{\Phi\left(y^{a}\right)}{r} S\left(z^{A}\right) \equiv \Omega\left(y^{a}\right) S\left(z^{A}\right)$, where $S\left(z^{A}\right)$ are the scalar spherical harmonics. For radial coordinate we sometimes utilize the tortoise coordinate $r_{*}$, defined via $\frac{d r_{*}}{d r}=\left(1-\frac{2 M}{r}\right)^{-1}$. The time dependence is taken to be $e^{-i \omega t}$. After simplification, Eq. (14) takes the following form:

$\frac{d^{2} \Phi}{d r_{*}^{2}}+\left(\omega^{2}-\tilde{V}\right) \Phi=0$

where

$$
\begin{aligned}
\tilde{V} & =V_{R W}+\frac{g(r)\left(1+f^{\prime}\right)}{3 f^{\prime \prime}} \\
& \equiv g(r)\left(\frac{k^{2}}{r^{2}}+\frac{2 M}{r^{3}}+\frac{1+f^{\prime}}{3 f^{\prime \prime}}\right) .
\end{aligned}
$$

This result for $f(R)=\alpha R^{2}$ theory was discussed in Ref. [53].

It is well known that each component of a tensor behaves differently under rotation group. As a result, the effective energy momentum tensor can be separated into scalar, vector and the tensor modes [59-61], as follows:

$$
\begin{aligned}
\delta T_{\mu \nu}^{e f f}= & {\left[\begin{array}{c|c}
\tau_{a b} \mathbf{S} & r \tau_{a}^{(S)} \mathbf{S}_{\mathbf{B}} \\
\hline r \tau_{a}^{(S)} \mathbf{S}_{\mathbf{B}} & r^{2} \delta P \gamma_{A B} \mathbf{S}+r^{2} \tau_{T}^{(S)} \mathbf{S}_{A B}
\end{array}\right] } \\
& +\left[\begin{array}{c|c}
0 & r \tau_{a}^{(V)} \mathbf{V}_{\mathbf{B}} \\
\hline r \tau_{a}^{(V)} \mathbf{V}_{\mathbf{B}} & r^{2} \tau_{T}^{(V)} \mathbf{V}_{A B}
\end{array}\right] \\
& +\left[\begin{array}{c|c}
0 & 0 \\
\hline 0 & r^{2} \Theta_{T} \mathbf{T}_{A B}
\end{array}\right]
\end{aligned}
$$

where $\mathbf{S}, \mathbf{V}_{\mathbf{A}}$ and $\mathbf{T}_{\mathbf{A B}}$ are the pure scalar, vector and tensor spherical harmonics, respectively. Rest of the tensors are defined from the pure spherical harmonic tensors. Further details, including the expression for $\Theta_{T}$ can be found in Refs. [59-61] and in Appendix A.
We find the various components of the energy momentum tensor from Eq. (20) to be

$$
\begin{aligned}
\tau_{a b}= & -\frac{f^{\prime \prime}}{\kappa^{2}\left(1+f^{\prime}\right)} \\
& \times\left[\bar{g}_{a b}\left(\tilde{\square} \Omega-\frac{k^{2} \Omega}{r^{2}}+\frac{2}{r} D^{a} r D_{a} \Omega\right)-D_{a} D_{b} \Omega\right] \\
\tau_{a}^{(S)}= & -\frac{f^{\prime \prime} k}{\kappa^{2}\left(1+f^{\prime}\right)} \bar{D}_{a}\left(\frac{\Omega}{r}\right) \\
\tau_{T}^{(S)}= & \frac{f^{\prime \prime} k^{2}}{\kappa^{2}\left(1+f^{\prime}\right)}\left(\frac{\Omega}{r^{2}}\right) \\
\delta P= & -\frac{f^{\prime \prime}}{\kappa^{2}\left(1+f^{\prime}\right)}\left(\tilde{\square}-\frac{k^{2}}{2 r^{2}}+\frac{1}{r} \bar{D}^{a} r \bar{D}_{a}\right) \Omega
\end{aligned}
$$

where $\delta R=\Omega\left(x^{a}\right) S\left(z^{A}\right)$.

The scalar and vector master equations get modified as follows:

$$
\begin{aligned}
\frac{d^{2} \Phi_{S}}{d r_{*}^{2}}+\left(\omega^{2}-V_{S}\right) \Phi_{S} & =S_{S}^{e f f}, \\
\frac{d^{2} \Phi_{V}}{d r_{*}^{2}}+\left(\omega^{2}-V_{V}\right) \Phi_{V} & =0,
\end{aligned}
$$

where

$$
\begin{aligned}
S_{S}^{e f f}= & -\frac{\beta}{H^{2} r^{4}}\left[\Omega \left\{2 ( 2 M - r ) \left(H r^{2}\left(-H+k^{2}-2\right)\right.\right.\right. \\
& \left.\left.+2 H M r+12 M^{2}\right)+2 H r^{5} \omega^{2}+P_{1} r^{2}(r-2 M)\right\} \\
& +2 r(r-2 M)\left\{2\left(-(H-6) M r+H r^{2}-12 M^{2}\right) \Omega^{\prime}\right. \\
& \left.\left.+H r^{2}(r-2 M) \Omega^{\prime \prime}\right\}\right],
\end{aligned}
$$

and

$$
\begin{aligned}
H & \equiv k^{2}-2+\frac{6 M}{r}, \\
P_{1} & \equiv-\frac{48 M^{2}}{r^{2}}+\frac{4 M}{r}\left(8-k^{2}\right)+2 k^{2}\left(k^{2}-2\right) .
\end{aligned}
$$

It should be noted that as $\beta \rightarrow 0$ these equations, appropriately, reduce to their GR counterparts even if the theory is $f(R)$ with $\beta=0$.

We also find that the tensor mode gives rise to the following equation [61],

$$
\tilde{\square} \Phi_{T}-\frac{V_{T}}{g} \Phi_{T}=0,
$$

where $\tilde{\square}$ represents the $\left(r_{*}-t\right)$ part of the d'Alembertian operator and

$V_{T}=\frac{g}{r^{2}}\left[\lambda_{L}-2+\frac{2 M}{r}\right]$.

Above, $\lambda_{L}$ is the eigenvalue of the Lichnerowicz operator [61]. Therefore we see that the tensor and the vector 
modes do not undergo any modification. This is understandable because the effective energy-momentum tensor arises from the different combinations of the derivatives of the Ricci scalar. It has been shown in Ref. [68] that the tensor harmonic functions are identically zero in transverse-traceless (TT) gauge. For this reason we will not pursue the study of the tensor mode any further.

Since the background in our studies is a $R=0$ solution, its metric does not have any extra hairs. However, we notice from Eq. (22) that even though the background black hole metric has no additional hair and is similar to the black holes in GR, at the level of perturbations the Zerilli mode gets modified from GR due to an extra source term. This source term originates because at the level of perturbations the extra massive mode that was absent in the background now gets excited. The excited massive mode "generates" hair via $\beta$. This tells us that the perturbations will therefore get modified from GR. As a result, we can expect that the detection of QNMs through gravitational wave observations will, in principle, provide us with the opportunity to distinguish between GR and $f(R)$ theories of gravity.

\section{Solution for QNM}

\subsection{Solution in radiation zone}

As we can see from the structure of the equations, the scalar equation gets modified by a source term. Therefore, it is understandable that the solution of that equation will have two parts: (a) One of these will be for the homogeneous part of the equation; this is identical to the GR solution; (b) The second will be the solution of the inhomogeneous part. Solution of the vector equation will be identical to the GR solution. Therefore, we can write

$\Phi_{S}=\Phi_{S}^{G R}+\Phi_{S}^{I H}, \quad \Phi_{V}=\Phi_{V}^{G R}$.

This implies that from the observational perspective the nonzero inhomogeneous part will be the signature of any deviation from GR.

If we take the $r \rightarrow \infty$ limit, then we find that

$\left.S_{S}^{e f f}\right|_{r \rightarrow \infty}=-\frac{2 \beta}{H}\left[\omega^{2} \Phi+\Phi^{\prime \prime}\right]$,

where $H=k^{2}-2$. However, due to Eq. (18),

$\frac{d^{2} \Phi}{d r^{* 2}}=\left(\frac{1}{3 \beta}-\omega^{2}\right) \Phi$.

The source term becomes

$\left.S_{S}^{e f f}\right|_{r \rightarrow \infty}=-\frac{2}{3 H} \Phi$.
Equation (30) has solution of the form $e^{i \chi r_{*}}$, with

$\chi^{2}=\omega^{2}-\frac{1}{3 \beta}$.

From Eq. (22) we find,

$\left.\Phi_{S}\right|_{r \rightarrow \infty}=e^{i \omega r_{*}}-\frac{2 \beta}{H} e^{i \chi r_{*}}$.

This gives the form of the inhomogeneous term arising due to the $f(R)$ theory:

$\Phi_{S}^{I H}=-\frac{2 \beta}{H} e^{i \chi r_{*}}$.

Therefore, one can see that there is a deviation from GR in this $f(R)$ theory, howsoever tiny its effect might be on observables.

In Eq. (33) we derived the asymptotic deviation of the $f(R)$ QNMs from GR. This provides the opportunity to constrain $f(R)$ theories by using it. In principle, there exists the possibility of bounding $\beta$ by observationally tracking how much energy is lost by QNMs and if that deviates from the prediction of GR. Solar system tests have already constrained $f(R)$ theories (details can be found in Appendix E). In comparison, the results found in the current work lend themselves to possible tests using $\mathrm{GW}$ observations. The inhomogeneous term arises due to the existence of the massive polarization mode, as seen from Eqs. (15) and (17). This occurs because the scalar mode and the massive mode are coupled, as was shown in Eq. (22). Through this inhomogeneous term, energy transfer can occur between the massive mode and the scalar mode. Therefore, tests for non-GR polarizations and energy content in each polarization may, in principle, constrain these $f(R)$ theories.

Another observational aspect of these findings is the breaking of iso-spectrality. It has been shown in Ref. [49] that the scalar and vector modes have identical spectra in GR. But now due to the source term it becomes evident that in general $f(R)$ theories this iso-spectrality will not be satisfied. The homogeneous equation will have an iso-spectral solution to the vector mode but the inhomogeneous part will not be iso-spectral. This point has been discussed in Sect. 5.2. Reference [53] claimed that the breaking of iso-spectrality will be there for all $f(R)$ theories. We prove above that in general the iso-spectrality will be violated if the theory has nonzero $\beta$. The details of the breakdown of the iso-spectrality has been discussed in 5.2.

\subsection{Broken iso-spectrality}

Breaking of iso-spectrality between the scalar and vector modes in the context of $\alpha R^{2}$ theory has already been discussed in Ref. [53]. Even though they claim it to be a universal phenomenon for $f(R)$ theories, we showed above that 
this is not true unless $\beta \neq 0$. For this reason we will investigate for the first time in detail exactly how iso-spectrality breaks down when $\beta=0$.

Iso-spectrality in GR was first discovered by Chandrasekhar [49]. We will follow his methods for this investigation. Say, $Z_{1,2}$ satisfy the following equations w.r.t. $r_{*}$ :

$$
\begin{aligned}
& \frac{d^{2} Z_{1}}{d r_{*}^{2}}+\omega^{2} Z_{1}=V_{1} Z_{1}, \\
& \frac{d^{2} Z_{2}}{d r_{*}^{2}}+\omega^{2} Z_{2}=V_{2} Z_{2},
\end{aligned}
$$

where $V_{1}$ and $V_{2}$ are the corresponding potentials. Using the ansatz that $Z_{1}=p Z_{2}+q Z_{2}^{\prime}$ and taking its derivative twice it is possible to show

$$
\begin{aligned}
Z_{1}^{\prime \prime}= & {\left[p^{\prime \prime}+\left(p+2 q^{\prime}\right)\left(V_{2}-\omega^{2}\right)+q V_{2}^{\prime}\right] Z_{2} } \\
& +\left[p^{\prime}+q\left(V_{2}-\omega^{2}\right)+p^{\prime}+q^{\prime \prime}\right] Z_{2}^{\prime},
\end{aligned}
$$

where the prime denotes derivative w.r.t. $r_{*}$. Comparing it with Eq. (35) Chandrasekhar showed that

$$
\begin{aligned}
& q\left(V_{1}-V_{2}\right)=2 p^{\prime}+q^{\prime \prime}, \\
& p\left(V_{1}-V_{2}\right)=p^{\prime \prime}+2 q^{\prime}\left(V_{2}-\omega^{2}\right)+q V_{2}^{\prime} .
\end{aligned}
$$

Now, finding a solution for $p$ and $q$ establishes a relation between $Z_{1}$ and $Z_{2}$. For the QNMs of the Schwarzschild solution in GR it is possible to identify, $Z_{1}=\Phi_{S}, Z_{2}=$ $\Phi_{V}, V_{1}=V_{S}$ and $V_{2}=V_{V}$. It is also possible to find $p$ and $q$. As a result, one can show that

$$
\begin{aligned}
\Phi_{S / V} & =\frac{1}{\frac{-\lambda^{2}(\lambda+1)^{2}}{9 M^{2}}-\omega^{2}}\left(\mp W \Phi_{V / S}+\frac{d \Phi_{V / S}}{d r_{*}}\right), \\
W & =\frac{6 M(2 M-r)}{r^{2}(6 M+2 \lambda r)}-\frac{\lambda(\lambda+1)}{3 M},
\end{aligned}
$$

where $\lambda=\frac{(l-1)(l+2)}{2}$. Due to this relationship it becomes evident that if $\Phi_{S}$ depends on the radial coordinate as $e^{i \omega r_{*}}$ then so does $\Phi_{V}$. Therefore, they share the same spectrum [69].

By inspecting Eq. (35) it is clear that the reason for such a simplification is its homogeneous nature. If we modify Eq. (35) with a source term, as in

$$
\begin{aligned}
& \frac{d^{2} Z_{1}}{d r_{*}^{2}}+\omega^{2} Z_{1}=V_{1} Z_{1}+S, \\
& \frac{d^{2} Z_{2}}{d r_{*}^{2}}+\omega^{2} Z_{2}=V_{2} Z_{2},
\end{aligned}
$$

then an ansatz of the previous form, $Z_{1}=p Z_{2}+q Z_{2}^{\prime}$, again leads to

$$
\begin{aligned}
Z_{1}^{\prime \prime}= & {\left[p^{\prime \prime}+\left(p+2 q^{\prime}\right)\left(V_{2}-\omega^{2}\right)+q V_{2}^{\prime}\right] Z_{2} } \\
& +\left[p^{\prime}+q\left(V_{2}-\omega^{2}\right)+p^{\prime}+q^{\prime \prime}\right] Z_{2}^{\prime} .
\end{aligned}
$$

But comparison with Eq. (39) does not lead to equations of $p, q$ that are independent of $Z_{1}$. So, in the sourced case it is not possible to have $Z_{1}=p Z_{2}+q Z_{2}^{\prime}$ as a solution. This eventually results in the violation of iso-spectrality.

One key point is worth mentioning. It is always possible to separate $Z_{1}$ as $Z_{1}=Z_{1}^{G R}+Z_{1}^{I H}$, where

$$
\frac{d^{2} Z_{1}^{G R}}{d r_{*}^{2}}+\omega^{2} Z_{1}^{G R}=V_{1} Z_{1}^{G R}
$$

and

$\frac{d^{2} Z_{1}^{I H}}{d r_{*}^{2}}+\omega^{2} Z_{1}^{I H}=V_{1} Z_{1}^{I H}+S$.

Therefore, it is possible to have $Z_{1}^{G R}=p Z_{2}+q Z_{2}^{\prime}$ and, consequently, there will exist an iso-spectral solution. Hence, $\Phi_{S}$ in $f(R)$ theories will have a part that is iso-spectral to $\Phi_{V}$ and another part that arises solely due to the source term that does not obey iso-spectrality.

\subsection{Massive mode}

As already shown above in Eq. (14) there exists a massive mode in $f(R)$ theories. The equation for this scalar mode is exactly equivalent to the equation of a scalar field around a Schwarzschild black hole in GR. The QNM of a massive scalar field around a Schwarzschild black hole in GR has been studied extensively [70,71]. Because of the similarity between the two problems, the results for the QNM frequency of the massive mode relevant for our current work will be exactly the same as the ones found in those works. Naturally, the QNM frequency values found in those works depend on the mass of the scalar field. In our current work that mass depends on the specific $f(R)$ theory chosen. Even though there are stringent restrictions on the value of this mass from solar system observations (see Appendix E), it is possible that independent restriction may be found from GW observations. Current constraints on the mass imply that if an $f(R)$ theory is the correct theory of gravity then, $m \geq 1.25 \times 10^{4} \mathrm{~m}^{-1}$.

One key point is worth mentioning here. Usually $\omega$ is a complex number, which implies that $\chi$ should be such a number as well. Consequently, the QNMs are damped. On top of that, the large value of the mass of the massive mode will give rise to a strong damping pattern. As a result, the contribution of the inhomogeneous part in Eq. (33) will be very less. But massive scalar fields in Schwarzschild backgrounds can have purely real $\omega$ [72]. This originates mainly from the sub-dominant asymptotic contribution arising due to the irregular singularity at infinity [72]. These modes are called quasi-resonance frequency modes [71]. The requirement for the existence of such modes is $\omega_{Q R M}<m$ [72], where $m$ is the mass of the massive mode. Further numerical study revealed that mass has a crucial role in the damping of 
that mode. They showed that the greater the mass the lesser is the damping rate. Therefore, purely real modes originate corresponding to the non-damping oscillation. They also showed that for a given mass of the field a certain number of lower overtones disappear. However, this disappearance happens only for the lower overtones, while the remaining overtones are still damped. Hence, owing to the non-zero mass of the massive mode the inhomogeneous term will contain some quasi-resonance contribution that will not decay as fast as the other damped overtones.

\section{Connecting with scalar-tensor theories}

It has been shown in several works that there is an equivalence between $f(R)$ theories and Brans-Dicke scalar-tensor theories (STT) [30,73-77]. In this section we investigate what implication can be found from such equivalence for a perturbed Schwarzschild black hole. For this reason first we will discuss the perturbation equations in STT around a Schwarzschild black hole in Einstein frame. Then we discuss the connection between the QNMs in $f(R)$ theory and STT.

The Einstein equations in STT can be written as,

$G_{\mu \nu}=R_{\mu \nu}-\frac{1}{2} R g_{\mu \nu}=T_{\mu \nu}^{S T T}$,

where,

$T_{\mu \nu}^{S T T}=\kappa^{2}\left[\nabla_{\mu} \phi \nabla_{\nu} \phi-g_{\mu \nu}\left(\frac{1}{2} g^{\alpha \beta} \nabla_{\alpha} \phi \nabla_{\beta} \phi+V(\phi)\right)\right]$,

and the scalar field $\phi$ satisfies,

$\square \phi-V^{\prime}(\phi)=0$.

In this work we focus on the perturbation of a hairless Schwarzschild BH. Therefore, the LHS of Eq. (43) is zero for the background. This implies that for the background one has $\phi=\phi_{C}$, where $\phi_{C}$ is some constant value of the scalar field $\phi$ that satisfies $V\left(\phi_{C}\right)=0$.

The perturbed part of Eq. (43) takes the following form,

$\delta G_{\mu \nu}=\delta T_{\mu \nu}^{S T T}=-\left.\kappa^{2} \bar{g}_{\mu \nu} V^{\prime}(\phi)\right|_{\phi=\phi_{C}} \delta \phi$,

where $\delta \phi$ is the perturbation in scalar field $\phi$.

For simplicity we will write $\left.V^{\prime}(\phi)\right|_{\phi=\phi_{C}}$ as $V^{\prime}\left(\phi_{C}\right)$. To isolate the dynamic part of the perturbation we separate $\delta \phi$ in orbit space and angular space, as has been described earlier. For that purpose we write $\delta \phi=\frac{\Phi^{S T T}\left(y^{a}\right)}{r} S\left(z^{A}\right) \equiv$ $\Omega^{S T T}\left(y^{a}\right) S\left(z^{A}\right)$, where $S\left(z^{A}\right)$ are the scalar spherical harmonics. For radial coordinate we use the tortoise coordinate $\left(r_{*}\right)$. The time dependence is taken to be $e^{-i \omega t}$. After simpli- fication, Eq. (45) takes the following form:

$\frac{d^{2} \Phi^{S T T}}{d r_{*}^{2}}+\left(\omega^{2}-\tilde{V}\right) \Phi^{S T T}=0$,

where

$\tilde{V}=g(r)\left(\frac{k^{2}}{r^{2}}+\frac{2 M}{r^{3}}+V^{\prime \prime}\left(\phi_{C}\right)\right)$.

The scalar and vector master equations get modified as follows:

$$
\begin{aligned}
& \frac{d^{2} \Phi_{S}}{d r_{*}^{2}}+\left(\omega^{2}-V_{S}\right) \Phi_{S}=S_{S}^{S T T}=-2 g(r) \frac{\kappa^{2}}{H} V^{\prime}\left(\phi_{C}\right) \Phi^{S T T} \\
& \frac{d^{2} \Phi_{V}}{d r_{*}^{2}}+\left(\omega^{2}-V_{V}\right) \Phi_{V}=0
\end{aligned}
$$

where

$H \equiv k^{2}-2+\frac{6 M}{r}$.

Details of the calculation can be found in the Appendix C.

\subsection{Radiation zone solution and broken iso-spectrality}

As we can see from the structure of the equations, the scalar equation gets modified by a source term. Therefore, it is understandable that the solution of that equation will have two parts: (a) One of these will be for the homogeneous part of the equation, and will be identical to the GR solution; (b) The second part will be the solution of the inhomogeneous part. The solution of the vector equation will be the same as the corresponding case in GR. Therefore, we can write

$\Phi_{S}=\Phi_{S}^{G R}+\Phi_{S}^{I H}, \quad \Phi_{V}=\Phi_{V}^{G R}$

From the observational perspective this implies that the nonzero inhomogeneous part is the signature of deviation from GR. If we take the $r \rightarrow \infty$ limit then we find that the source term becomes

$\left.S_{S}^{S T T}\right|_{r \rightarrow \infty}=-\frac{2 \kappa^{2} V^{\prime}\left(\phi_{C}\right)}{H} \Phi^{S T T}$,

where $H=k^{2}-2$.

Asymptotically Eq. (47) has solution of the form $e^{i \chi r_{*}}$ with

$\chi^{2}=\omega^{2}-V^{\prime \prime}\left(\phi_{C}\right)$.

Hence from Eq. (22) we find,

$\left.\Phi_{S}\right|_{r \rightarrow \infty}=e^{i \omega r_{*}}-\frac{2 \kappa^{2} V^{\prime}\left(\phi_{C}\right)}{H V^{\prime \prime}\left(\phi_{C}\right)} e^{i \chi r_{*}}$. 
Therefore, the inhomogeneous term arising in the STT:

$\Phi_{S}^{I H}=-\frac{2 \kappa^{2} V^{\prime}\left(\phi_{C}\right)}{H V^{\prime \prime}\left(\phi_{C}\right)} e^{i \chi r_{*}}$.

Therefore, we find that there is a deviation from GR in STT, as is manifest when comparing with Eq. (28), howsoever tiny the effect of this term might be on observables. Therefore, in STT also the iso-spectrality is broken as long as $V^{\prime}\left(\phi_{C}\right) \neq 0$.

\subsection{Equivalence with $f(R)$}

In this section we will discuss the correspondence between the QNMs of a Schwarzschild BH in STT and those of such a BH in $f(R)$ theory. Let us begin by considering the $f(R)$ gravity model (known as Jordan frame) that has a Lagrangian of the following form:

$$
\begin{aligned}
S_{J} & =\frac{1}{2 \kappa^{2}} \int d^{4} x \sqrt{-g} f(R), \\
& =\int d^{4} x \sqrt{-g}\left(\frac{f^{\prime}(R) R}{2 \kappa^{2}}-\frac{f^{\prime}(R) R-f(R)}{2 \kappa^{2}}\right),
\end{aligned}
$$

where $f(R)$ is the same as that used in Eq. (1); we will retain this definition until Eq. (61) below. Conformal transformation of the Jordan frame metric yields the new metric $\boldsymbol{g}_{\mu \nu}=\Omega^{2} g_{\mu \nu}$, where $\Omega$ is the conformal factor. Under the identification

$\Omega^{2}=f^{\prime}(R), \quad \kappa \phi=\sqrt{\frac{3}{2}} \ln f^{\prime}(R)=\sqrt{\frac{3}{2}} \ln \Omega^{2}$,

the action reduces to

$S_{E}=\int d^{4} x \sqrt{-\boldsymbol{g}}\left(\frac{\boldsymbol{R}}{2 \kappa^{2}}-\frac{1}{2} \boldsymbol{g}^{\mu \nu} \nabla_{\mu} \phi \nabla_{\nu} \phi-V(\phi)\right)$,

which is known as the Einstein-frame action; here, the potential $V(\phi)$ can be written as

$V(\phi)=\frac{f^{\prime}(\phi) R(\phi)-f(\phi)}{2 \kappa^{2} f^{\prime}(\phi)^{2}}$.

In our convention, all the metric dependent quantities that originate from a conformal transformation of the Jordan frame are boldfaced. The field equation for $\boldsymbol{g}_{\mu \nu}$ can be found by varying the Einstein frame action, and is given by [73,85]

$$
\begin{aligned}
\boldsymbol{G}_{\mu \nu} & \equiv \boldsymbol{R}_{\mu \nu}-\frac{1}{2} \boldsymbol{R} \boldsymbol{g}_{\mu \nu} \\
& =\kappa^{2}\left[\nabla_{\mu} \phi \nabla_{\nu} \phi-\boldsymbol{g}_{\mu \nu}\left(\frac{1}{2} \boldsymbol{g}^{\alpha \beta} \nabla_{\alpha} \phi \nabla_{\beta} \phi+V(\phi)\right)\right] .
\end{aligned}
$$

In the current work our objective is to compare the QNMs in these two theories. The background metrics are taken to be identical. In general, however, it is not possible to have identical background in both the theories that are related to each other through Eq. (58). We now return to the usage $f(R) \rightarrow R+f(R)$, as has been described in Sect. 2. For
Schwarzschild solution in $f(R)$ we find $\Omega^{2}=1+f^{\prime}(0)$ from Eq. (58). For both of the theories to have the same metric as conformally related solutions we need $\Omega^{2}=1$, in case of Swarzschild solution it is achievable only if $f^{\prime}(0)=0$. This is not true in general. Hence, in general, a Schwarzschild solution in $f(R)$ theory does not conformally transform to a Schwarzschild solution in STT, even though it is equivalent to some solution in STT. Therefore, QNMs in $f(R)$ theory do not conformally transform to QNMs of the same background in STT in general.

This property is not limited to $R=0$ solutions alone. For a general background, one has $\Omega^{2}=1+f^{\prime}(R)$, and the two conformally related theories can have identical metric solutions if and only if $f^{\prime}(R)=0$. If this condition is not satisfied then even though the corresponding theories are conformally equivalent to each other, they can not have an identical metric solution that are conformally related.

As we are discussing QNMs of Schwarzschild BHs, we will focus on the case $\Omega^{2}=1+f^{\prime}(0)$. When $f^{\prime}(0)=0$ a Schwarzschild solution in $f(R)$ theory corresponds to a Schwarzschild solution in the corresponding STT. For this reason we will focus on this particular category. In this case $\Omega^{2}=1$ for the background. Therefore, for perturbation we can write

$$
\begin{aligned}
\kappa(\bar{\phi}+\delta \phi) & =\sqrt{\frac{3}{2}} \ln \left(1+f^{\prime \prime}(0) \delta R\right) \\
& =\sqrt{\frac{3}{2}} f^{\prime \prime}(0) \delta R=\sqrt{\frac{3}{2}} f^{\prime \prime}(0) \frac{\Phi\left(y^{a}\right)}{r} S\left(Z^{A}\right),
\end{aligned}
$$

where $\delta \phi$ and $\delta R$ are perturbed scalar field in STT and perturbed Ricci scalar in $f(R)$ theory, respectively. If we separate out the angular dependence as $\delta \phi=\Phi^{S T T}\left(y^{a}\right) S\left(Z^{A}\right) / r$, then we can identify

$\Phi^{S T T}=\sqrt{\frac{3}{2 \kappa^{2}}} f^{\prime \prime}(0) \Phi\left(y^{a}\right)$.

From Eq. (62), we see that in STT the background scalar field, $\bar{\phi}=0$. Similarly in $f(R)$ theory extra scalar mode is not present. Therefore, the extra scalar mode gets excited in the presence of a perturbation; otherwise it remains at zero. Vector QNMs are identical to GR in both the theories. Therefore, the vector mode in $f(R)$ theory is equivalent to the vector mode in STT; in fact, they are identical.

As has already been discussed, the scalar QNM in both the theories have a homogeneous part that is iso-spectral to the vector QNM and an inhomogeneous part that is not isospectral to the vector QNM. The homogeneous part in both the theories is identically equal to the GR QNM. Hence, the homogeneous part of the scalar mode in $f(R)$ theory is equivalent to the homogeneous part of the scalar mode in STT: they are identical. 
The difference arises in the inhomogeneous part. This difference can be understood by computing $\delta V(\phi)$,

$$
\begin{aligned}
\delta V(\phi)= & \frac{\bar{R} f^{\prime \prime}(\bar{R}) \delta R}{2 \kappa^{2}\left(1+f^{\prime}(\bar{R})\right)^{2}} \\
& -\frac{\left(1+f^{\prime}(\bar{R})\right) \bar{R}-(\bar{R}+f(\bar{R}))}{\kappa^{2}\left(1+f^{\prime}(\bar{R})\right)^{3}} f^{\prime \prime}(\bar{R}) \delta R,
\end{aligned}
$$

where $\delta R$ can be represented in terms of $\delta \phi$ from Eq. (62). For $\bar{R}=0$ this gives $\frac{d V(\phi)}{d \phi}=0$. Hence, the source term in the Eq. (49) vanishes for STT, leading to a vanishing inhomogeneous solution. The Regge-Wheeler and Zerilli mode in these STTs are identical to GR.

This can be understood further with an example, namely that of the $f(R)=\alpha R^{2}$ theory. The conformal transformation can be expressed as follows:

$\Omega^{2}=1+2 \alpha R, \quad \kappa \phi=\sqrt{\frac{3}{2}} \ln (1+2 \alpha R)$.

The potential of the corresponding STT can be written as

$V(\phi)=\frac{\left(1-e^{-\sqrt{2} \kappa \phi / \sqrt{3}}\right)^{2}}{8 \alpha \kappa^{2}}$.

A Schwarzschild solution for this theory has $\phi_{C}=0$. Using it in Eq. (66) we find $\left.\frac{d V(\phi)}{d \phi}\right|_{\phi=0}=0$. Hence, there are no source terms in Eq. (49), thereby making it identical to GR. On the other hand, if we take $f(R)=\alpha R^{2}$ in Eq. (22), we find that $\beta=2 \alpha$, which leads to a non-vanishing source term. Therefore, the QNMs in that theory are different from those in GR, and isospectrality is violated in this example of $f(R)$. But in the corresponding STT the Zerilli QNM is exactly like GR and the iso-spectrality stays unbroken.

This result indicates some interesting features of the correspondence between $f(R)$ theory and STT. We have shown explicitly that the iso-spectrality breaking is not present in the QNMs after one transforms to the Einstein frame using a conformal transformation. A particular metric solution of an $f(R)$ theory gets mapped to some metric solution of the conformally related scalar-tensor theory. This by itself does not, however, imply that the two spacetime solutions are identical; therefore, it is not necessary for them to share all of the same characteristics. Indeed, in general, a particular solution in $f(R)$ will not get mapped to exactly the same solution in the conformally related scalar-tensor theory. This is often the case when the conformal factor is not unity. In that case there is no obvious reason for their QNMs to share similar properties. What we have shown here is that when an $f(R)$ there and an STT both have the Schwarzschild metric as solution and conformal factor relating these respective solutions is unity, then isopectrality is not broken for the Schwarzschild QNMs in STT even though it is broken for the Schwarzschild QNMs in $f(R)$.

\section{Discussion}

In this work we have focused on the QNMs of a Schwarzschild black hole in a general $f(R)$ theory that allows the $R=0$ solution. We came to the conclusion that in general $f(R)$ theories the iso-spectrality between the scalar and the vector mode will not be valid. Breaking of iso-spectrality was discussed in the work [53] for the specific case of $\alpha R^{2}$ theory. They claimed this result will be true for all other theories also. We found that this claim is not entirely true. In general, there will be breaking of isospectrality, but it is possible to have theories where it is not true, such as when $f(R)=\alpha R^{3}$. Indeed, it is possible to conclude that the isospectrality will be violated if $f^{\prime \prime} /\left(1+f^{\prime}\right) \neq 0$.

Secondly, we discussed that the structure of QNMs gets modified at the asymptote. This modification arises as an inhomogeneous term alongside the GR contribution. Therefore, not only the iso-spectrality will be violated but also the QNM structure will get modified. But for $f^{\prime \prime} /\left(1+f^{\prime}\right)=0$, this contribution along with the breaking of the isospectrality will be absent, resulting in zero deviation from GR in first order perturbation. This does not imply that the ringdown spectrum will be exactly like GR. If we take second order perturbation then we find,

$$
\begin{aligned}
\delta^{2} G_{\mu \nu}= & \frac{\mathcal{S}_{\mu \nu}}{1+f^{\prime}} \\
\mathcal{S}_{\mu \nu}= & f^{\prime \prime} \delta R \delta R_{\mu \nu}+f^{\prime \prime \prime} \bar{g}_{\mu \nu} \delta R \bar{\square} \delta R \\
& +f^{\prime \prime} \delta g_{\mu \nu} \square \delta R+f^{\prime \prime} \bar{g}_{\mu \nu} \delta(\bar{\square} \delta R)+f^{\prime \prime \prime} \frac{\bar{R}}{4} \bar{g}_{\mu \nu} \delta R^{2} \\
& -f^{\prime \prime} \frac{\bar{g}_{\mu \nu}}{4} \delta R^{2}+f^{\prime \prime} \frac{\bar{R}}{4} \delta g_{\mu \nu} \delta R+f^{\prime \prime} \frac{\bar{R}}{4} \bar{g}_{\mu \nu} \delta^{2} R \\
& -f^{\prime \prime} \delta\left(\nabla_{\mu} \nabla_{\nu} \delta R\right)-f^{\prime \prime \prime} \delta R \nabla_{\mu} \nabla_{\nu} \delta R \\
& +\left(f-R f^{\prime}\right) \frac{\delta^{2} g_{\mu \nu}}{2} .
\end{aligned}
$$

For $\bar{R}=0$ solution $\mathcal{S}$ reduces to,

$$
\begin{aligned}
\mathcal{S}_{\mu \nu}= & f^{\prime \prime} \delta R \delta R_{\mu \nu}+f^{\prime \prime \prime} g_{\mu \nu} \delta R \square \delta R+f^{\prime \prime} \delta g_{\mu \nu} \square \delta R \\
& +f^{\prime \prime} g_{\mu \nu} \delta(\square \delta R)-f^{\prime \prime} \frac{g_{\mu \nu}}{4} \delta R^{2} \\
& -f^{\prime \prime} \delta\left(\nabla_{\mu} \nabla_{\nu} \delta R\right)-f^{\prime \prime \prime} \delta R \nabla_{\mu} \nabla_{\nu} \delta R .
\end{aligned}
$$

As it depends on $f^{\prime \prime \prime}$, the ringdown mode will contain information of the theory but the first order QNM will be exactly like GR. Therefore a detailed numerical relativity evolution will capture these deviations. As a result one can in principle constrain $\beta$ and $\left.\frac{d^{n} f(R)}{d R^{n}}\right|_{\bar{R}=0}$ solely from GW observations.

One key-point is worth mentioning here. In our current work we have focused only on the perturbation around a vacuum $\bar{R}=0$ solution. We have used these similar assumptions while interpreting the solar system test. Owing to this choice 
we have not been able to pursue the study of Chameleon screening in $f(R)$ theories [82]. This screening behaviour is the origin of the different behaviour of the theory at different scales. Due to the screening effect the mass of the massive scalar mode depends on the mass density of energy momentum tensor $\left(T_{\mu \nu}\right)$ in the space-time. Effective mass of the massive scalar mode becomes heavier in the region with high mass density and lighter in the low density region. Therefore, in different environments the mass of the massive scalar mode will have different values depending on the density profile. This gives rise to the screening mechanism. Therefore, interpreting and using the solar system constraints should be done carefully. But unfortunately due to vacuum assumption $\left(T_{\mu \nu}=0\right)$, we have not been able to explore this sector in our current work. Hence, a rigorous analysis with non-vacuum condition is an important endeavour that may be taken up in the future.

QNMs in scalar-tensor theories have recently been calculated in Refs. [55,56,79-81]. It has been shown before that a class of scalar-tensor theories are equivalent to $f(R)$ theories. Tattersall et al. have also found results similar the ones noted here in the context of scalar-tensor theories. Due to the equivalence of scalar-tensor theories and $f(R)$ theories this is, of course, expected. The equivalence between Brans-Dicke with $\omega_{0}=0$ and $f(R)$ theory is valid under the condition $f^{\prime \prime}(R) \neq 0$ (related to the sufficient condition for invertibility between the two theories). When $f^{\prime \prime}$ is not defined, or vanishes, the equality $\phi=f^{\prime}(R)$ and the equivalence between the two theories cannot be guaranteed, although this is not a priori excluded by $f^{\prime \prime}=0$ [73]. Therefore, there are families of $f(R)$ that in principle are not equivalent to scalar-tensor theories. Interestingly, in our work we see that this is precisely the sector where $f(R)$ does not bring any modification w.r.t. GR, mainly due to the vanishing coupling between the GR mode and the extra massive scalar mode.

Acknowledgements We thank Sumanta Chakraborty, S. Shankaranarayanan and Varun Sahni for the useful inputs. This work is supported in part by the Navajbai Ratan Tata Trust. SD would like to thank University Grants Commission (UGC), India, for financial support as senior research fellow.

Data Availability Statement This manuscript has no associated data or the data will not be deposited. [Authors' comment: This work has not used any data].

Open Access This article is licensed under a Creative Commons Attribution 4.0 International License, which permits use, sharing, adaptation, distribution and reproduction in any medium or format, as long as you give appropriate credit to the original author(s) and the source, provide a link to the Creative Commons licence, and indicate if changes were made. The images or other third party material in this article are included in the article's Creative Commons licence, unless indicated otherwise in a credit line to the material. If material is not included in the article's Creative Commons licence and your intended use is not permitted by statutory regulation or exceeds the permit- ted use, you will need to obtain permission directly from the copyright holder. To view a copy of this licence, visit http://creativecomm ons.org/licenses/by/4.0/.

Funded by $\mathrm{SCOAP}^{3}$.

\section{Appendix A: Spherical harmonics}

Any field on a spherically symmetric background can be classified as a scalar, vector and tensor depending on their transformation under the rotation group. For this reason three kinds of spherical harmonics are used to separate out the angular dependence.

One subset of these functions are the scalar spherical harmonics. Any scalar function on a spherically symmetric spacetime is expressible as a sum of the scalar spherical harmonics, $Y_{l m}(\theta, \phi)$. We call them $S\left(Z^{A}\right)$.

A vector field $U_{A}$ can be expressed as, $U_{A}=V_{A}+D_{A} S$, where $S$ is the scalar spherical harmonic defined above. $V_{A}$ are called the vector spherical harmonics.

A tensor field $X_{A B}$ on spherically symmetric spacetime can be expressed as,

$X_{A B}=T_{A B}+2 D_{(A} V_{B)}+\hat{L}_{A B} S+\gamma_{A B} S$,

where $\gamma_{A B}$ is the metric on the two sphere. $V_{A}$ and $S$ are the vector and scalar spherical harmonics, respectively. $\hat{L}_{A B}$ is the Lichnerowicz operator defined as, $\hat{L}_{A B}=D_{A} D_{B}-$ $\frac{1}{2} \gamma_{A B} . S, V_{A}$ and $T_{A B}$ are called the pure spherical harmonic functions.

They satisfy the following equations,

$$
\begin{aligned}
\left(\hat{\square}+k^{2}\right) S & =0, \\
\left(\hat{\square}+k^{2}\right) V_{A} & =0, \\
\left(\hat{\square}+k^{2}\right) T_{A B} & =0,
\end{aligned}
$$

where $k^{2}=l(l+1)$, and $l$ is a non-negative integer.

From the pure spherical harmonics several useful vector and tensor fields can be defined. We only mention a few among them, namely,

$$
\begin{aligned}
S_{A} & =-\frac{1}{k} D_{A} S, \\
S_{A B} & =\frac{1}{k^{2}} D_{A} D_{B} S+\frac{1}{2} \gamma_{A B} S, \\
V_{A B} & =-\frac{1}{2 k}\left(D_{A} V_{B}+D_{B} V_{A}\right) .
\end{aligned}
$$

\section{Appendix B: Separation of effective energy momentum tensor}

Let us assume that the metric of a manifold is as follows,

$$
\begin{aligned}
d s^{2} & =g_{a b}(x) d x^{a} d x^{b}+r^{2}(x) d \Omega^{2}, \\
d \Omega^{2} & =\gamma_{A B} d z^{A} d z^{B} .
\end{aligned}
$$


In this case the Christoffel symbols take particular forms as discussed below. A hat represents connection on two sphere and ${ }^{2} \Gamma_{b c}^{a}$ represents the connection in the orbit space. The nonzero components of the connection are as follows,

$$
\begin{aligned}
\Gamma_{b c}^{a} & ={ }^{2} \Gamma_{b c}^{a}, \\
\Gamma_{B C}^{a} & =-r D^{a} r \gamma_{B C}, \\
\Gamma_{a B}^{A} & =\frac{D_{a} r}{r} \delta_{B}^{A}, \\
\Gamma_{B C}^{A} & =\hat{\Gamma}_{B C}^{A} .
\end{aligned}
$$

Using these results for connection we can calculate various covariant derivatives. Various components of double covariant derivatives of a scalar function $F\left(x^{a}, z^{A}\right)$ can be calculated to be as follows,

$$
\begin{aligned}
\nabla_{a} \nabla_{b} F & =D_{a} D_{b} F, \\
\nabla_{a} \nabla_{B} F & =r D_{a}\left(\frac{1}{r} D_{B} F\right), \\
\nabla_{A} \nabla_{b} F & =D_{A} D_{b} F-\frac{D_{b} r}{r} D_{A} F, \\
\nabla_{A} \nabla_{B} F & =D_{A} D_{B} F+r D^{a} r D_{a} F \gamma_{A B}, \\
\square F & =\tilde{\square} F+\frac{1}{r^{2}} \hat{\square} F+\frac{2}{r} D^{a} r D_{a} F .
\end{aligned}
$$

It has been shown that the Einstein equation gets modified in our case. As result we get an effective energy momentum tensor of the following form,

$$
\delta T_{\mu \nu}^{e f f}=-\frac{\beta}{\kappa^{2}}\left[\bar{g}_{\mu \nu} \bar{\square}-\bar{\nabla}_{\mu} \bar{\nabla}_{\nu}\right] \delta R .
$$

Since $\delta R$ is a scalar function, we write it as,

$$
\delta R=\Omega\left(x^{a}\right) S\left(z^{A}\right) .
$$

Now using the expressions in (B2) and (B3) in Eq. (B4) and using the separation explained in Eq. (20) we find,

$$
\begin{aligned}
\tau_{a b} & =-\frac{\beta}{\kappa^{2}}\left[\bar{g}_{a b}\left(\tilde{\square} \Omega-\frac{k^{2} \Omega}{r^{2}}+\frac{2}{r} D^{a} r D_{a} \Omega\right)-D_{a} D_{b} \Omega\right], \\
\tau_{a}^{(S)} & =-\frac{\beta}{\kappa^{2}} k \bar{D}_{a}\left(\frac{\Omega}{r}\right), \\
\tau_{T}^{(S)} & =\frac{\beta}{\kappa^{2}} k^{2}\left(\frac{\Omega}{r^{2}}\right), \\
\delta P & =-\frac{\beta}{\kappa^{2}}\left(\tilde{\square}-\frac{k^{2}}{2 r^{2}}+\frac{1}{r} \bar{D}^{a} r \bar{D}_{a}\right) \Omega .
\end{aligned}
$$

All other terms arising in Eq. (20) are zero.

$$
\begin{aligned}
\tau_{a b} & =-\bar{g}_{a b} \frac{d V}{d \phi} \frac{\Phi^{S T T}}{r} \\
\tau_{a} & =\tau_{T}^{(S)}=0 \\
r^{2} \delta P & =-\frac{d V}{d \phi} \frac{\Phi^{S T T}}{r} .
\end{aligned}
$$$$
\text { For STT } \delta T_{\mu \nu}=-\bar{g}_{\mu \nu} \frac{d V}{d \phi} \delta \phi \text {. }
$$

\section{Appendix C: Effective source}

The perturbation equations with a source term around the Schwarzschild metric was calculated in [59]. We take the appropriate limit i.e. $n=2$ and put electromagnetic perturbation to zero. After doing that the result found is,

$$
\begin{aligned}
S_{S}^{e f f}= & \frac{g \kappa^{2}}{r H}\left[-H S_{T}-\frac{P_{1} S_{t}}{i \omega H}-4 g \frac{r S_{t}^{\prime}}{i \omega}\right. \\
& \left.-4 r g S_{r}+\frac{P_{2} r S_{t}^{r}}{i \omega H}+2 r^{2} \frac{S_{t}^{r \prime}}{i \omega}+2 r^{2} S_{r}^{r}\right],
\end{aligned}
$$

where prime denotes radial derivative.

The functions in the previous equation are dependent on the effective energy momentum tensor and the metric in the following manner,

$$
\begin{aligned}
S_{a b} & =\tau_{a b}, \\
S_{a} & =\frac{r \tau_{a}}{k}, \\
S_{T} & =\frac{2 r^{2}}{k^{2}} \tau_{T}, \\
H & =k^{2}-2+\frac{6 M}{r}, \\
P_{1} & =-\frac{48 M^{2}}{r^{2}}+\frac{4 M}{r}\left(8-k^{2}\right)-2 k^{2}\left(k^{2}-2\right), \\
P_{2} & =\frac{24 M}{r} .
\end{aligned}
$$

After a little calculation it can be expressed as,

$$
\begin{aligned}
S_{S}^{e f f}= & -\frac{\beta}{H^{2} r^{4}}\left[\Omega \left\{2 ( 2 M - r ) \left(H r^{2}\left(-H+k^{2}-2\right)\right.\right.\right. \\
& \left.\left.+2 H M r+12 M^{2}\right)+2 H r^{5} \omega^{2}+P_{1} r^{2}(r-2 M)\right\} \\
& +2 r(r-2 M)\left\{2\left(-(H-6) M r+H r^{2}-12 M^{2}\right) \Omega^{\prime}\right. \\
& \left.\left.+H r^{2}(r-2 M) \Omega^{\prime \prime}\right\}\right]
\end{aligned}
$$

For STT,

$$
\begin{aligned}
S_{a b} & =\tau_{a b}, \\
S_{a} & =0, \\
S_{T} & =0 .
\end{aligned}
$$

$S_{S}^{S T T}=-\frac{2 g \kappa^{2}}{H} \frac{d V}{d \phi} \Phi^{S T T}$

\section{Appendix D: GW propagation in Minkowski background}

We take the opportunity of the current work to discuss the propagation of GW in Minkowski spacetime for general 
$f(R)$ theory of gravity. To study the propagation of GW in general $f(R)$ theory, in Minkowski background we take $\bar{g}_{\mu \nu}=\eta_{\mu \nu}$. As a result the perturbation equations become

$\delta G_{\mu \nu}^{e f f}=\left[\delta R_{\mu \nu}-\frac{1}{2} \eta_{\mu \nu} \delta R\right]-\frac{f(0)}{\left(1+f^{\prime}(0)\right)} \frac{h_{\mu \nu}}{2}$,

$\delta T_{\mu \nu}^{e f f}=-\frac{f^{\prime \prime}}{\kappa^{2}\left(1+f^{\prime}(0)\right)}\left[\eta_{\mu \nu} \bar{\square}-\partial_{\mu} \partial_{\nu}\right] \delta R(h)$.

Taking the trace of Eq. (11) it can be found that,

$$
\begin{aligned}
3 f^{\prime \prime} \partial_{\mu} \partial^{\mu} \delta R-\left(1+f^{\prime}(0)\right) \delta R & =0, \\
\square \delta R+m^{2} \delta R & =0,
\end{aligned}
$$

where $m^{2}=-\frac{\left(1+f^{\prime}\right)}{3 f^{\prime \prime}}=-\frac{1}{3 \beta}$. Owing to the real-valuedness of $m$, it must be the case that $\beta \leq 0[64,78]$. This implies that $\frac{1+f^{\prime}}{f^{\prime \prime}} \leq 0$.

Along the lines of Ref. [64] we define the infinitesimal Gauge transformation

$h_{\mu \nu}^{\prime}=h_{\mu \nu}-\left(\beta \delta R+\frac{h}{2}\right) \eta_{\mu \nu}$,

and choose the Lorentz gauge condition,

$\partial_{\mu} h^{\prime \mu \nu}=0$.

Defining $\delta G_{\mu \nu}^{e f f}-\kappa^{2} \delta T_{\mu \nu}^{e f f} \equiv \delta \mathcal{G}_{\mu \nu}$, it can be shown that

$\delta \mathcal{G}_{\mu \nu}=-\frac{1}{2} \square h_{\mu \nu}^{\prime}$

The equation $\delta \mathcal{G}_{\mu \nu}=0$ then implies

$\square h_{\mu \nu}^{\prime}=0$.

Therefore, we can see that the only difference the general $f(R)$ theory makes relative to the case studied in Ref. [64] is that the $a_{2}$ in their work gets replaced by $\beta=\frac{f^{\prime \prime}}{1+f^{\prime}}$.

\section{Appendix E: Constraints on $f(R)$}

In the work [64] different methods for constraining $f(R)$ models have been discussed. We will mainly focus on the fifth force test. For this reason it is important to take the Newtonian limit. The result will be similar to that found in Ref. [64] by replacing $\Upsilon$ with $m$. The metric then takes the form,

$$
\begin{aligned}
d s^{2}= & \left\{1-\frac{2 M}{r}\left[1+\frac{\exp (-m r)}{3}\right]\right\} d t^{2} \\
& -\left\{1+\frac{2 M}{r}\left[1-\frac{\exp (-m r)}{3}\right]\right\} d \Sigma^{2},
\end{aligned}
$$

where, $d \Sigma^{2}=d x^{2}+d y^{2}+d z^{2}$.

The structure of the metric [64] gives rise to the potential of Yukawa type, as was already discussed in [64]. In fifthforce tests such potential has been studied extensively, where the considered form is,

$V(r)=\frac{M}{r}\left[1+\alpha \exp \left(-\frac{r}{\lambda}\right)\right]$.

From the Eöt-Wash experiment $[83,84]$ it is possible to put strong constraint, $\lambda=m^{-1} \leq 8 \times 10^{-5} \mathrm{~m}$. Therefore, we are finding that for general $f(R)$ this puts up the bound $|\beta|=$ $\left|\frac{f^{\prime \prime}}{1+f^{\prime}}\right| \leq 2 \times 10^{-9} \mathrm{~m}^{2}$.

\section{References}

1. B.P. Abbott et al. (LIGO Scientific and Virgo Collaboration), Phys. Rev. Lett. 118, 221101 (2017)

2. B.P. Abbott et al. (LIGO Scientific and Virgo Collaboration), Phys. Rev. Lett. 116, 241103 (2016)

3. B.P. Abbott et al. (LIGO Scientific and Virgo Collaboration), Phys. Rev. X 6, 041015 (2016)

4. B.P. Abbott et al. (LIGO Scientific and Virgo Collaboration), Phys. Rev. Lett. 116, 061102 (2016)

5. B.P. Abbott et al. (LIGO Scientific and Virgo Collaborations), Phys. Rev. Lett. 119, 161101 (2017)

6. The LIGO Scientific Collaboration, the Virgo Collaboration, arXiv: 1805.11581

7. S. De, D. Finstad, J.M. Lattimer, D.A. Brown, E. Berger, C.M. Biwer arXiv:1804.08583

8. T. Zhao, J.M. Lattimer, arXiv: 1808.02858

9. E. Annala, T. Gorda, A. Kurkela, A. Vuorinen, Phys. Rev. Lett. 120, 172703 (2018)

10. D. Radice, A. Perego, F. Zappa, S. Bernuzzi, Astrophys. J. Lett. 852, L29 (2018)

11. B.P. Abbott et al. (LIGO Scientific and Virgo Collaboration), Phys. Rev. Lett. 118, 221101 (2017)

12. T. Baker, E. Bellini, P.G. Ferreira, M. Lagos, J. Noller, I. Sawicki, Phys. Rev. Lett. 119, 251301 (2017)

13. J.M. Ezquiaga, M. Zumalacárregui, Phys. Rev. Lett. 119, 251304 (2017)

14. L. Lombriser, A. Taylor, JCAP 03, 031 (2016)

15. L. Lombriser, N.A. Lima, Phys. Lett. B 765, 382-385 (2017)

16. B.P. Abbott et al. (LIGO Scientific and Virgo Collaboration), Phys. Rev. Lett. 116, 221101 (2016)

17. R. Konoplya, A. Zhidenko, Phys. Lett. B 756, 350 (2016)

18. P. Amaro-Seoane et al., arXiv: 1702.00786

19. M. Armano, H. Audley, G. Auger, J.T. Baird, M. Bassan, P. Binetruy, M. Born, D. Bortoluzzi, N. Brandt et al., Phys. Rev. Lett. 116, 231101 (2016)

20. P.A. Seoane, S. Aoudia, S. Babak, P. Binétruy, E. Berti, A. Bohé, C. Caprini, M. Colpi, N.J. Cornish, K. Danzmann, J.F. Dufaux, J. Gair, O. Jennrich, P. Jetzer, A. Klein, R.N. Lang, A. Lobo, T. Littenberg, S.T. McWilliams, G. Nelemans, A. Petiteau, E.K. Porter, B.F. Schutz, A. Sesana, R. Stebbins, T. Sumner, M. Vallisneri, S. Vitale, M. Volonteri, H. Ward, Class. Quantum Gravity 29(12), 124016 (2012)

21. S. Vitale, Gen. Relativ. Gravit. 46, 1730 (2014)

22. S. Babak, J. Gair, A. Sesana, E. Barausse, C.F. Sopuerta, C.P.L. Berry, E. Berti, P. Amaro-Seoane, A. Petiteau, A. Klein, Phys. Rev. D 95, 103012 (2017)

23. C.M. Will, Theory and Experiment in Gravitational Physics (Cambridge University Press, Cambridge, 1993)

24. C.M. Will, Living Rev. Relativ. 17, 4 (2014)

25. K.S. Stelle, Phys. Rev. D 16, 953 (1977)

26. K.S. Stelle, Gen. Relativ. Gravit. 9, 353 (1978)

27. B. Shahid-Saless, J. Math. Phys. 31, 242 (1990) 
28. R.H. Brandenberger, (1992). arXiv:gr-qc/9210014

29. R.H. Brandenberger, (1993). arXiv:gr-qc/9302014

30. S. Nojiri, S.D. Odintsov, Phys. Rep. 505, 59-144 (2011)

31. S. Nojiri, S.D. Odintsov, V.K. Oikonomou, Phys. Rep. 692, 1-104 (2017)

32. R.H. Brandenberger, V.F. Mukhanov, A. Sornborger, Phys. Rev. D 48, 1629 (1993)

33. M. Trodden, V.F. Mukhanov, R.H. Brandenberger, Phys. Lett. B 316, 483 (1993)

34. A.A. Starobinsky, Phys. Lett. B 91, 99 (1980)

35. W. Hu, I. Sawicki, Phys. Rev. D 76, 064004 (2007)

36. S. Nojiri, S.D. Odintsov, Phys. Rev. D 68, 123512 (2003)

37. G. Cognola, E. Elizalde, S. Nojiri, S.D. Odintsov, L. Sebastiani, S. Zerbini, Phys. Rev. D 77, 046009 (2008)

38. N.D. Birrell, P.C.W. Davies, Quantum Fields in Curved Spacetime (Cambridge University Press, Cambridge, 1982)

39. I.L. Buchbinder, S.D. Odintsov, I.L. Shapiro, Effective Actions in Quantum Gravity (IOP, Bristol, 1992)

40. G.A. Vilkovisky, Class. Quantum Gravity 9, 895 (1992)

41. R.A. Konoplya, A.F. Zinhailo, Z. Stuchlík, arXiv:1903.03483 [grqc]

42. A.F. Zinhailo, Eur. Phys. J. C 78, 992 (2018)

43. X. Zhang, Y. Ma, Phys. Rev. D 84, 064040 (2011)

44. G.J. Olmo, P. Singh, JCAP 01, 030 (2009)

45. T. Regge, J.A. Wheeler, Phys. Rev. 108, 1063 (1957)

46. F.J. Zerilli, Phys. Rev. Lett. 24, 737 (1970)

47. E. Newman, R. Penrose, J. Math. Phys. (N.Y.) 3, 566 (1962)

48. C.V. Vishveshwara, Nature (London) 227, 936 (1970)

49. S. Chandrasekhar, The Mathematical Theory of Black Holes (Oxford University Press, New Delhi, 2010)

50. H.P. Nollert, Class. Quantum Gravity 16, R159 (1999)

51. K.D. Kokkotas, B.G. Schmidt, Living Rev. Relativ. 2, 2 (1999)

52. R.A. Konoplaya, A. Zhidenko, Rev. Mod. Phys. 83, 793 (2011)

53. S. Bhattacharya, S. Shankaranarayanan, Phys. Rev. D 96, 064044 (2017)

54. S. Bhattacharyya, S. Shankaranarayanan, Eur. Phys. J. C 78, 737 (2018)

55. O.J. Tattersall, P.G. Ferreira, Phys. Rev. D 97, 104047 (2018)

56. O.J. Tattersall, P.G. Ferreira, M. Lagos, Phys. Rev. D 97, 044021 (2018)

57. R.P. Woodard, in 3rd Aegean Summer School: The Invisible Universe: Dark Matter and Dark Energy, ed. by L. Papantonopoulos, vol. 720 (Springer, Berlin, 2007), pp. 430-433

58. Y.S. Myung, T. Moon, E.J. Son, Phys. Rev. D 83, 124009 (2011)
59. A. Ishibashi, H. Kodama, Prog. Theor. Phys. Suppl. 189, 165 (2011)

60. H. Kodama, A. Ishibashi, O. Seto, Phys. Rev. D 62, 064022 (2000)

61. H. Kodama, A. Ishibashi, Prog. Theor. Phys. 110, 701 (2003)

62. U.H. Gerlach, U.K. Sengupta, Phys. Rev. D 19, 2268 (1979)

63. S. Capozziello, C. Corda, M.F. De Laurentis, Phys. Lett. B 669, 255 (2008)

64. C.P.L. Berry, J.R. Gair, Phys. Rev. D 83, 104022 (2011)

65. A. Nishizawa, A. Taruya, K. Hayama, S. Kawamura, M. Sakagami, Phys. Rev. D 79, 082002 (2009)

66. H.R. Kausar, L. Philippoz, P. Jetzer, Phys. Rev. D 93, 124071 (2016)

67. C. Corda, J. Cosmol. Astropart. Phys. 0704, 009 (2007)

68. R.G. Cai, L.M. Cao, Phys. Rev. D 88, 084047 (2013)

69. E. Berti, V. Cardoso, A.O. Starinets, Class. Quantum Gravity 26, 163001 (2009)

70. L.E. Simone, C.M. Will, Class. Quantum Gravity 9, 963-978 (1992)

71. A. Ohashi, M.A. Sakagami, Class. Quantum Gravity 21, 3973 (2004)

72. R.A. Konoplya, A.V. Zhidenko, Phys. Lett. B 609, 377-384 (2005)

73. T.P. Sotiriou, V. Faraoni, Rev. Mod. Phys. 82 (2010)

74. A. De Felice, S. Tsujikawa, Living Rev. Relativ. 13, 3 (2010). https://doi.org/10.12942/lrr-2010-3

75. S. Capozziello, R. de Ritis, A.A. Marino, Class. Quantum Gravity 14, 3243-3258 (1997)

76. R. Catena, M. Pietroni, L. Scarabello, Phys. Rev. D 76, 084039 (2007)

77. T. Chiba, M. Yamaguchi, J. Cosmol. Astropart. Phys. 2013, 40 (2013)

78. H.-J. Schmidt, Astro. Nachr. 307, 339 (1986)

79. Tsutomu Kobayashi, Hayato Motohashi, Teruaki Suyama, Phys. Rev. D 85, 084025 (2012)

80. A. Ganguly, R. Gannouji, M. Gonzalez-Espinoza, C. PizarroMoya, Class. Quantum Gravity 35, 145008 (2018)

81. Tsutomu Kobayashi, Hayato Motohashi, Teruaki Suyama, Phys. Rev. D 89, 084042 (2014)

82. C. Burrage, J. Sakstein, Living Rev. Relativ. 21, 1 (2018)

83. D.J. Kapner, T.S. Cook, E.G. Adelberger, J.H. Gundlach, B.R. Heckel, C.D. Hoyle, H.E. Swanson, Phys. Rev. Lett. 98, 021101 (2007)

84. C.D. Hoyle, D.J. Kapner, B.R. Heckel, E.G. Adelberger, J.H. Gundlach, U. Schmidt, H.E. Swanson, Phys. Rev. D 70, 042004 (2004)

85. S. Chakraborty, S. SenGupta, Eur. Phys. J. C 76, 552 (2016). https:// doi.org/10.1140/epjc/s10052-016-4394-0 